\title{
Variaciones alométricas durante el crecimiento en siete especies de garzas (Aves: Ardeidae)
}

\author{
Dennis Denis Ávila \\ Calle 25 entre J e I, Vedado, Habana, Cuba. Dpto. Biología Animal y Humana, Facultad Biología, Universidad de La \\ Habana; dda@fbio.uh.cu
}

Recibido 24-III-2017. Corregido 20-VII-2017. Aceptado 21-VIII-2017.

\begin{abstract}
Alometric variations during growth in seven species of egrets and herons (Aves: Ardeidae). The order Ciconiiformes include wading bird species of sizes from medium to high, with body growth dynamic that can be analyzed to enlighten micro evolutionary trends. Egrets and herons (family Ardeidae) evolved from a common ancestor, but there are differences in adult body shapes, and their evolution has been suggested to be based on heterochronic processes. However, previous researches on growth have focused only in lineal dimension, and alometric changes have not been studied. In the current paper I described changes in body proportions during growth in seven ardeid species, and analyzed body growth under a phylogenetic point of view, to identify the primitive morphology pattern among genus Butorides and Nycticorax. For this purpose, I calculated bill/ tarsus rate in 353 nestlings, measured between 1998 and 2006, and assessed their changes with age and body weight. All species showed marked differences in proportion changes extension between hatching and an analogous growth moment, except Bubulcus that grows almost isometrically. Alometric changes during growth and at hatch, generate a differential growth that produced the different adult morphologies expressed among egrets and herons. The general trends were toward a slight increase in the middle of the growth period up to a lowering to almost the same initial proportions. Growth in the first life stages tends to be more isometric and differences get higher latter in growth. The hypothesis of Nycticorax as peramorphic morfotype is more parsimonious with changes trends in the group, resulting in a relative extremities extension with positive alometry in bill and tarsus in all species. This hypothesis is consistent with a gradual hipermorphosis that reaches a maximum expression in Ardea. Rev. Biol. Trop. 65 (4): 1347-1357. Epub 2017 December 01.
\end{abstract}

Key words: alometry, development, wading bird, microevolution, heterochrony.

Las dinámicas del crecimiento corporal, incluyendo la velocidad del crecimiento y la forma de las curvas de incremento de las dimensiones de cada parte del cuerpo, son características inherentes de cada especie con un componente de modulación ambiental determinado (Ricklefs, 1979a) y han sido una línea clásicamente estudiada dentro de la Ornitología (Kushlan, 1977; Ricklefs, 1973, 1979b; O'Connor 1984; Freymann \& Schuchmann, 2008). La ecomorfología de las aves ha sido bien estudiada, por las estrechas relaciones entre los patrones morfológicos y las distintas líneas ecológicas relacionadas con la alimentación, uso del hábitat o relaciones inter e intraespecíficas. En el grupo de las aves acuáticas, la familia Ardeidae contiene un grupo de especies genéricamente conocidas como garzas, que han sido estudiadas en varias oportunidades en relación al crecimiento corporal (Siegfried, 1973; Hudson, Dawson, \& Hill, 1974; Custer \& Peterson, 1991; Denis, 2011). En Cuba existen 12 especies de esta familia y de ellas se han estudiado aspectos de la alimentación (Acosta, Mugica, \& Martínez, 1990), morfometría (Denis, Mugica, \& Acosta 2000), uso del hábitat (Acosta, Mugica, \& Denis, 2002; Mugica, Acosta, \& Denis, 2003) y reproducción (Denis, Rodríguez, Rodríguez, \& Jiménez, 2003; Denis \& Ponce de León 2006; 
Denis, Rodríguez, Antunez, \& Fortes, 2009). Este grupo es reconocible por sus biotipos característicos de zancudas, identificable por la longitud de sus patas, cuellos y picos, como respuesta adaptativa a explotar los ecosistemas de humedales someros a través del vadeo (caminar dentro del agua). Por su relativa constancia en la forma, es un grupo modelo que se ha prestado para el análisis de los patrones de crecimiento de distintas partes corporales (Wersckul, 1979).

Denis (2011) observó que en ese grupo de aves existe la tendencia a que el patrón de crecimiento de cada variable corporal (pico, tarso y peso) pueda ser descrito, en mayor o menor medida, por las mismas ecuaciones de crecimiento. Esto puede estar relacionado con su evolución a partir de una condición plesiomórfica (morfología de un ancestro primitivo común), como respuesta a la presión selectiva relacionada con el uso del hábitat en ecosistemas de humedales. En este y en otros estudios previos se ha sugerido la posibilidad de que la evolución del grupo haya sido dirigida por cambios morfológicos heterocrónicos, aunque Cobo et al. (2000) señalaron también una posible base histológica alternativa, a partir del aumento de las tallas celulares. La heterocronía es un mecanismo evolutivo que aparece cuando hay cambios uniformes en las velocidades o cronología de algún proceso ontogénico, sin cambio en la estructura interna de este (Rice, 1997). Denis (2011) describe una relación entre el incremento de las estructuras corporales y la extensión temporal del proceso de crecimiento, y sugiere que esto apoya la hipótesis de una microevolución del grupo por una hipermorfosis (desarrollo excesivo de una estructura morfológica) causada por el retardo del momento final del crecimiento.

Sin embargo, los estudios realizados hasta ahora en las zancudas se han enfocado exclusivamente en aspectos absolutos del crecimiento (Kushlan, 1977; Custer \& Peterson, 1991; Denis 2011). Estudios alométricos, que son los que se enfocan en las variaciones conjuntas de diferentes estructuras, son mencionados únicamente a través de algunas proporciones o índices relativos, pero no se ha abordado toda la gama de transformaciones espaciales de la morfología corporal durante el crecimiento (Raff, 1996). Los cambios en las formas o proporciones corporales quedan, por esta razón, enmascarados por el efecto de las diferencias en las dimensiones lineales.

En la familia Ardeidae se han sugerido varias hipótesis filogenéticas a partir de hibridación de ADN (Sheldon, 1987), a partir de caracteres osteológicos (McCraken \& Sheldon, 1998) y por comparación del ADN del citocromo b (Sheldon et al., 2000). Todas coinciden en que el grupo proviene de un tronco común y que luego se divide en dos subgrupos: el primero con las especies del género Egretta y el segundo con las del género Ardea y Bubulcus. De forma independiente, en todas las filogenias aparecen Butorides y Nycticorax como los géneros más primitivos, sin poder resolverse cuál de los dos se relaciona más con el ancestro común del grupo, siendo posibles dos hipótesis: que la forma del ancestro fuese más parecida a la de Butorides o que fuese más parecida a la de Nycticorax. El estudio comparativo de los patrones de crecimiento y de la alometría durante el crecimiento podría arrojar luz sobre estas, teniendo en cuenta el principio de Huxley de que el desarrollo ontogenético recapitula la filogenia.

Por las razones anteriormente planteadas, en el presente trabajo se caracterizan los cambios en las proporciones corporales durante el crecimiento de siete especies de garzas, como vía para la identificación del morfotipo plesiomórfico más parsimonioso, atribuible al ancestro del grupo de las garzas, según la comparación de las proporciones de los pichones al nacer, en momentos análogos del crecimiento y en los individuos adultos.

\section{MATERIALES Y MÉTODOS}

Para describir el cambio de forma durante el crecimiento se tomaron los pesos y las dimensiones del pico y el tarso, al nacer y en el decimosexto día de edad, a 353 pichones de siete especies de garzas (Fig. 1). Los tamaños 


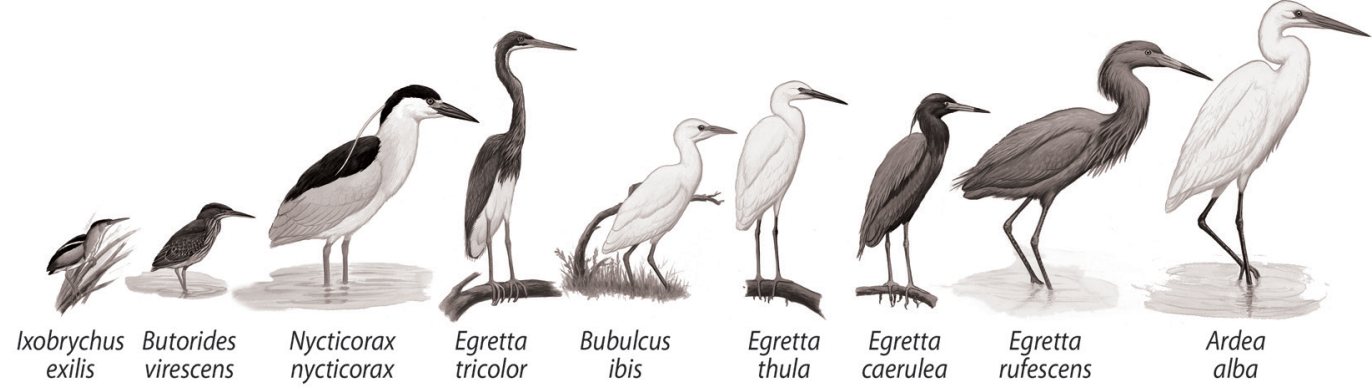

Fig. 1. Representantes de la familia Ardeidae, que contiene un conjunto de aves acuáticas reconocibles por su peculiar morfotipo, similares superficialmente pero con proporciones lineales distintas y marcadas diferencias en tallas.

Fig. 1. Representatives of Ardeidae family, that include a group of water birds easily recognized by its peculiar morphology, slightly resembled but with different proportions and marked differences in sizes.

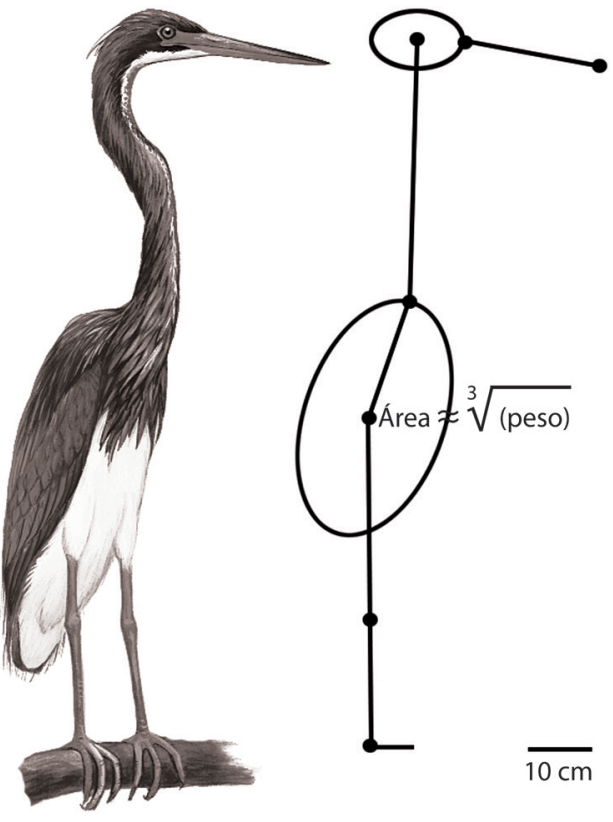

Fig. 2. Esquematización de las proporciones corporales en los adultos de las especies de garzas analizadas.

Fig. 2. Squematizing adult body proportions in the species of egrets and herons analyzed.

de muestra por especie fueron: 111 pichones de Bubulcus ibis, 77 de Egretta thula, 14 de E. alba, 10 de E. rufescens, 70 de E. tricolor, 49 de Butorides virescens y 22 de Nycticorax nycticorax. Estas medidas fueron obtenidas en pichones marcados y seguidos durante su crecimiento, en las colonias de la ciénaga de Birama
(Cuba), en el sistema de colonias de la Laguna Las Playas entre 1998 y 2006, y fueron empleadas por Denis (2011) (ver el artículo referido para otros detalles de su método de obtención).

Se calculó el índice largo del pico/largo del tarso, para cada individuo-edad y se comparó en el momento de la eclosión y en el momento del desarrollo, en el cual se alcanzaba el $30 \%$ de la longitud adulta del tarso. Este momento fue diferente para cada especie: a los cinco días de edad en B. ibis, a los siete días en Butorides y Nycticorax, a los nueve días en Egretta tricolor, a los diez en E. rufescens y a los doce días en Ardea alba. La alometría del índice fue descrita a partir de su comportamiento con el incremento del peso corporal.

A partir de las mediciones se confeccionaron modelos esquemáticos de las proporciones en cada especie (Fig. 2), que sirvieron de base para diagramas transformacionales simplificados de D`Arcy Thompson (o rejillas de distorsión). Thompson (1942) demostró que muchas alteraciones en la forma de especies relacionadas se deben a simples alteraciones en las escalas de los ejes cartesianos sobre los que se grafica la forma del cuerpo y desarrolló el método de representación que emplea una rejilla cuadrada confeccionada con las proporciones de una forma de referencia para superponerla a una nueva forma y distorsionar sus lados hasta que se ajustan, con lo cual 
se hacen evidentes las transformaciones de las formas. De las mismas fuentes bibliográficas mencionadas en el trabajo previo, se tomaron los valores morfométricos reportados para los adultos de cada especie. En estos esquemas simplificados, la longitud del pico y del tarso se representó de forma tal que estuviesen en la misma escala. El peso se representó a través del área de la elipse del cuerpo, con la cual se vinculó por una función raíz cuadrada, con un coeficiente de valor constante. A fin de simplificar el modelo gráfico, la tibia se representó verticalmente y su longitud se asumió igual a la del tarso, a pesar de que en los adultos tiende a ser como promedio un $30 \%$ superior, excepto en Ixobrychus exilis donde es solo un $16 \%$ mayor (datos no publicados). El cuello se representó de la misma longitud que la suma de los segmentos que representaban la tibia y el tarso, para simular el mantenimiento del centro de gravedad del cuerpo (aunque en los individuos adultos esta dimensión es generalmente entre un $30 \%$ y un $56 \%$ superior, y se compensa por las diferencias en pesos). Para eliminar el efecto de la talla general, todos los esquemas se superpusieron y se escalaron de forma isométrica, hasta que coincidieran las áreas de la elipse central del cuerpo.

Los diagramas transformacionales se confeccionaron para cada especie en la etapa adulta y en el primer día de nacido, tomando como morfotipo primitivo las proporciones de B. virescens y de N. nycticorax. Las variaciones alométricas durante el crecimiento dentro de cada especie, se graficaron con las medidas morfométricas cada tres días y tomando como configuración de referencia, las proporciones del pichón recién eclosionado. Para comparar, de forma estandarizada, las diferencias alométricas en el crecimiento entre especies, se hicieron los diagramas en dos porcentajes comunes del crecimiento (15 y $30 \%$ del desarrollo físico, medido por el peso corporal). Se asumió un crecimiento isométrico del tarso y el cuello (no comprobado pero necesario para conservar el centro de gravedad del cuerpo).

\section{RESULTADOS}

Al comparar entre especies la magnitud de cambios en las proporciones entre las longitudes del pico y el tarso mostraron diferencias muy marcadas entre el inicio del crecimiento postnatal y un momento análogo del crecimiento (Fig. 3). En Butorides este cambio fue ligeramente superior que en Nycticorax, y relativamente similar al que experimentan las garzas de mayor tamaño (Ardea y E. rufescens). De las garzas medianas, E. tricolor fue la que presentó el cambio mayor de todas las especies, consistente con su morfotipo más estilizado. En el mismo periodo de crecimiento, E. thula mostró un cambio muy pequeño entre las proporciones, mientras que el extremo lo constituyó Bubulcus, en la cual el cambio es prácticamente nulo, lo cual indica que ambas estructuras tienden a crecer de forma casi isométrica. Estas diferencias en las tasas relativas de crecimiento fueron consistentes con la ecología trófica y el uso del hábitat de estas especies, con cambios menores las especies menos especializadas en la captura de peces y presas móviles.

Los cambios alométricos durante el crecimiento, en relación a las proporciones de los pichones al nacer, genera el proceso de crecimiento diferencial que produce, a partir de estados iniciales de tamaños y proporciones físicas muy similares, las disímiles morfologías adultas que se expresan en el grupo de las garzas (Fig. 4). Las curvas de crecimiento lineal de las estructuras, expresadas en función de la edad sugirieron este comportamiento cuando fueron representadas en una misma escala, por las diferencias en sus formas, pero sin cuantificarlas explícitamente el análisis del cambio en las proporciones es de difícil aprehensión.

$\mathrm{Al}$ analizar las tendencias de cambios alométricos en la proporción pico tarso en estas especies, se pudieron observar tres tendencias generales (Fig. 5). En Nycticorx y Butorides, las especies más primitivas, se produjo una disminución de la proporción en la primera mitad del incremento de peso, que generalmente se produce entre la segunda y tercera semanas de vida, para luego incrementarse hasta valores 


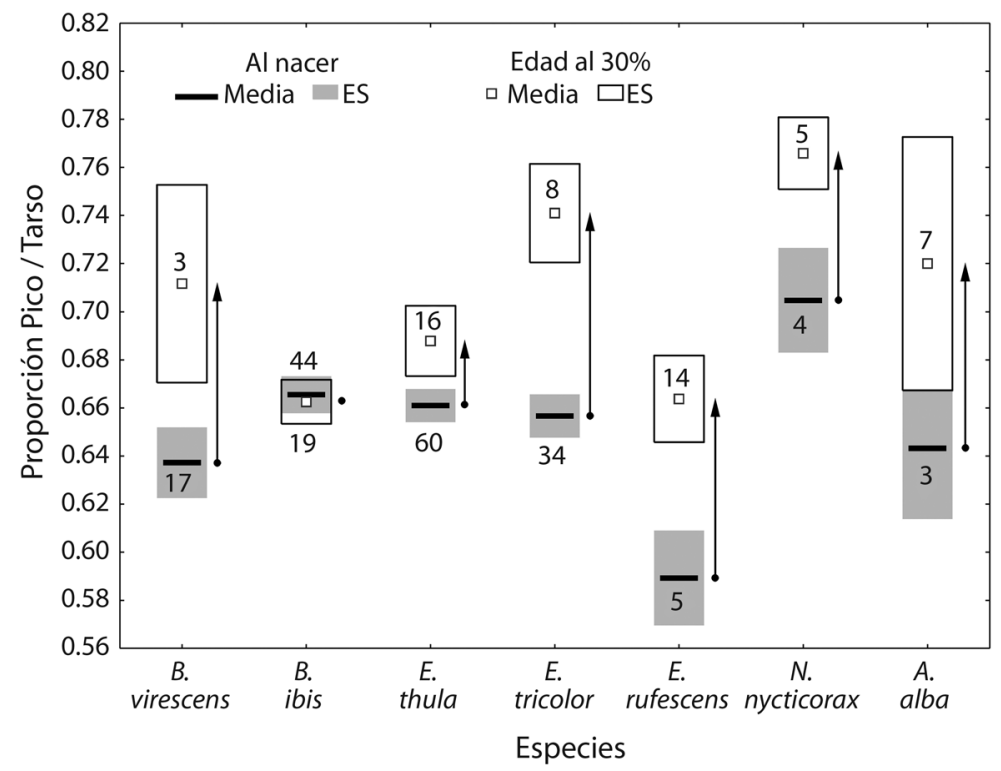

Fig. 3. Variación de la proporción longitud del pico / longitud del tarso al nacer y al alcanzar el 30 \% de la longitud del tarso en siete especies de garzas (Aves: Ardeidae).

Fig. 3. Variation in the rate bill length/ tarsus length at hatch and arriving to $30 \%$ of adult tarsus length in seven species of egrets and herons (Aves: Ardeidae).

muy superiores al del momento de la eclosión. En Bubulcus, E. thula, y Ardea la tendencia fue a una disminución progresiva suave en esta proporción. Y finalmente en E. tricolor, y en menor medida en E. rufescens, se produjo un aumento de la proporción en el periodo medio del crecimiento. La tendencia general, si se agrupan los datos de todas las especies, fue de un incremento ligero en el medio del crecimiento para luego descender a casi la misma proporción inicial. Estos patrones deben ser interpretados cuidadosamente, como comportamiento de índices relativos que son, ya que un mismo resultado puede provenir de dos cambios diferentes de las variables involucradas: tanto un aumento desmedido del pico como una disminución del tarso, pueden compensarse y producir el mismo valor de cambio del índice. Independientemente de esto, la conclusión general es innegable: las dinámicas relativas de crecimiento de picos y tarsos, en referencia al crecimiento en peso corporal, fueron diferentes y su comportamiento fue especie específico.
La similitud funcional de las dinámicas de crecimiento, una vez eliminadas las diferencias temporales y de asíntotas, es un elemento importante a favor de la semejanza estructural en el proceso de morfogénesis del biotipo de zancuda en las aves vadeadoras. A la luz de esta similitud, la relación directa entre la extensión temporal del proceso de crecimiento en estas especies, que produce distintos incrementos en las estructuras corporales adultas, apoya la hipótesis de que el cambio fundamental en la microevolución del pico y del tarso en la familia Ardeidae fue una hipermorfosis, causada por la extensión del periodo de crecimiento. Las marcadas diferencias específicas en la alometría del crecimiento de ambas estructuras y sus dinámicas de cambio con el aumento del peso corporal también son aspectos que apoyan esta idea.

Para analizar la evolución del grupo de las garzas a partir de los patrones de crecimiento a la luz de esta explicación evolutiva, se parte de las dos hipótesis en relación al morfotipo 

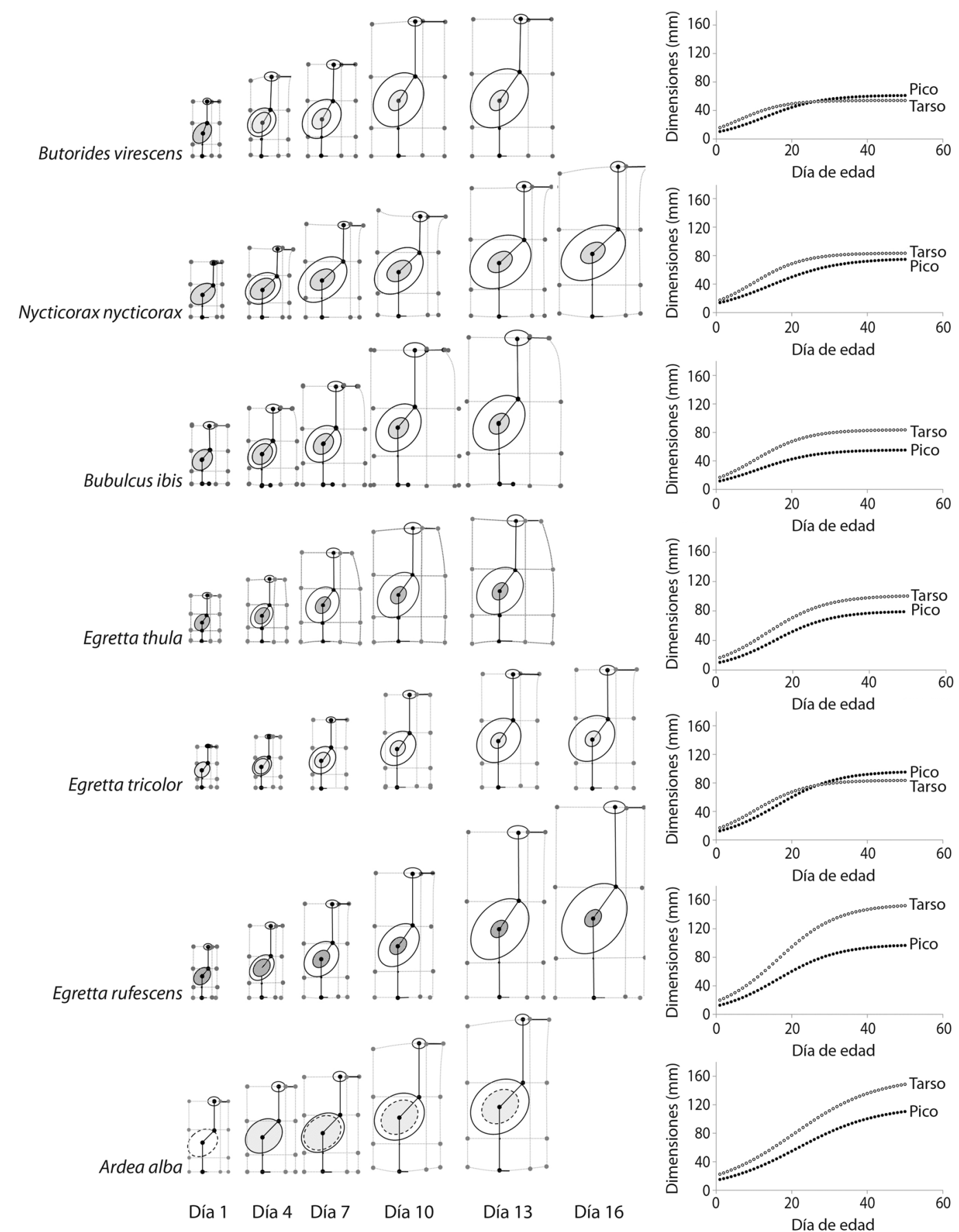

Fig. 4. Cambios alométricos durante el crecimiento en siete especies de garzas (Aves: Ardeidae). La rejilla de referencia es la del pichón recién eclosionado, así como el óvalo sombreado (excepto en $A$. alba donde no existen datos de masa al nacer y se tomó la masa del día 4). Las curvas de crecimiento del pico y el tarso fueron obtenidas por las ecuaciones de Denis (2011). Fig. 4. Alometric changes during growth in seven species of egrets and herons (Aves: Ardeidae). Reference grid is the one of the hatchling, as well as the shaded circle (but in $A$. alba where there is no data of mass at hatch and we use the mass at day 4). Growth curve of bills and tarsus were obtained from equations given by Denis (2011). 

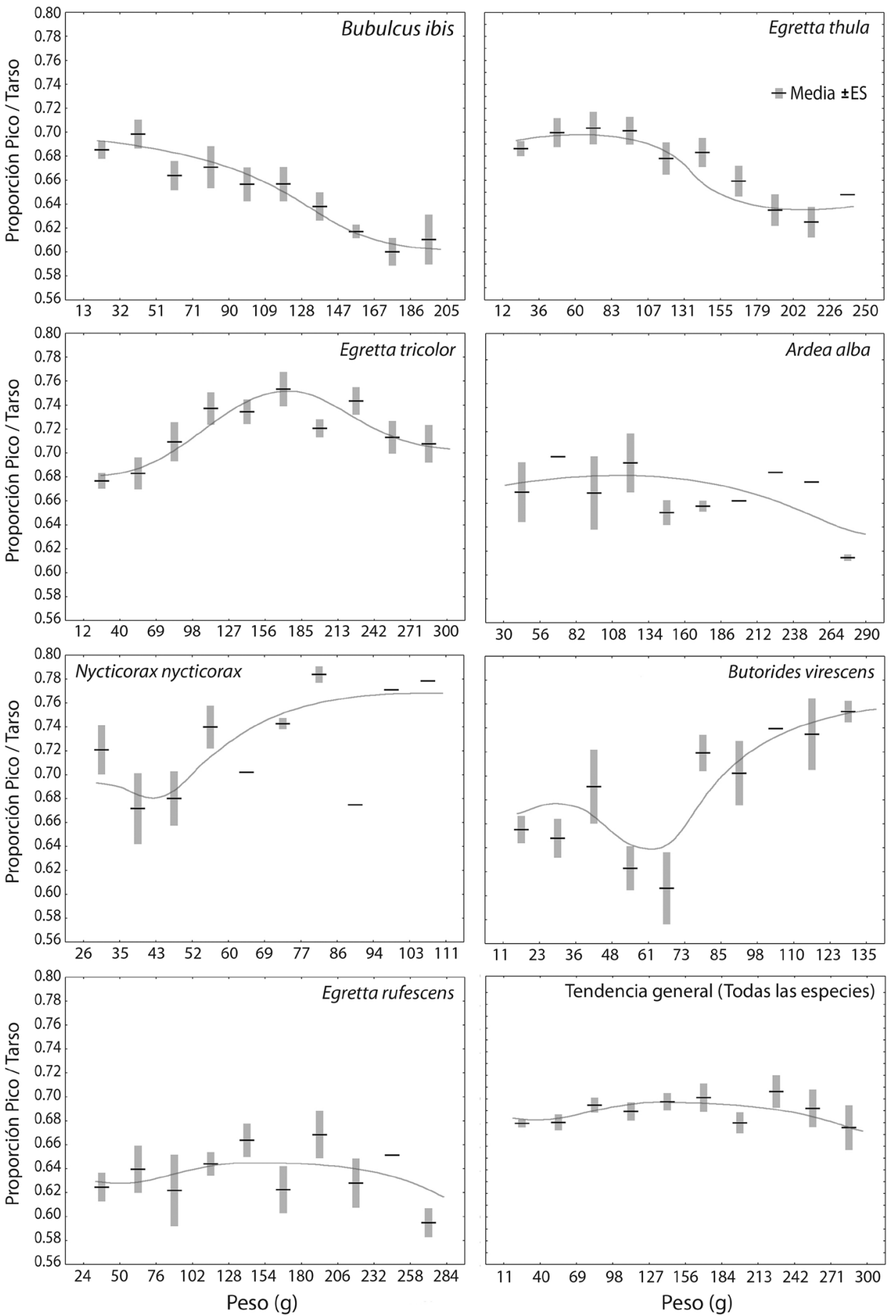

Fig. 5. Variación alométrica de la proporción longitud del pico / longitud del tarso durante el crecimento en siete especies de garzas (Aves: Ardeidae).

Fig. 5. Alometric variations of rate bill length/ tarsus length during growth in seven species of egrets and herons (Aves: Ardeidae). 


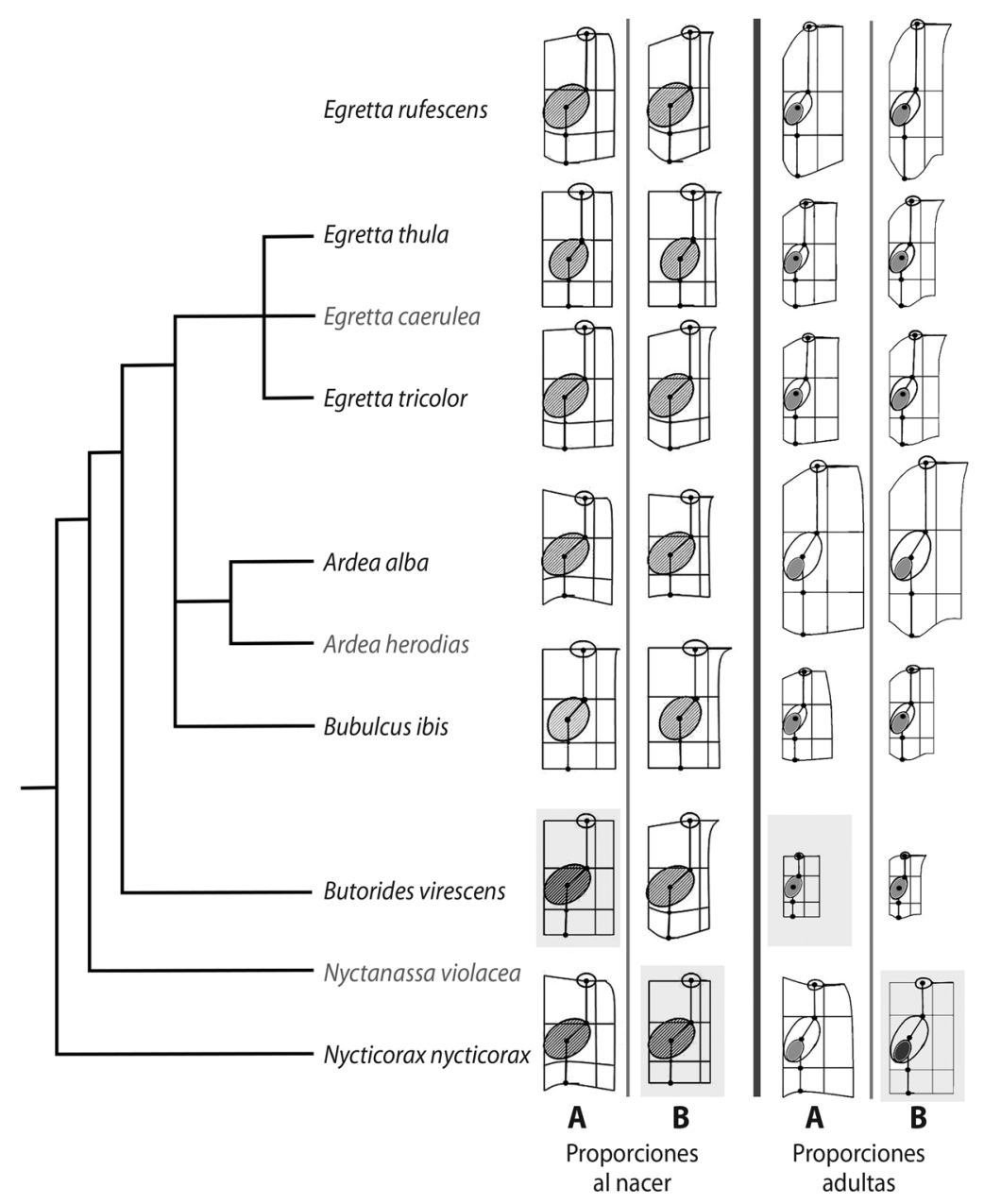

Fig. 6. Representación de las diferencias en proporciones corporales al eclosionar y en la fase adulta en siete especies de garzas, en relación a las hipótesis de la morfología primitiva, siguiendo el árbol filogenético propuesto por McCraken y Sheldon (1998) según caracteres osteológicos. A) Condición plesiomórfica similar a la forma actual de Butorides virescens; B) Condición plesiomórfica similar a la forma de Nycticorax nycticorax. El área del elipsoide central sin relleno en los adultos está en proporción directa a la raíz cúbica del peso corporal y el elipsoide sombreado representa la talla de la especie menor. Todas las dimensiones lineales están en la misma escala (aproximadamente 1: 94).

Fig. 6. Representation of differences in body proportions at hatch and adult states in seven species of egrets and herons, in relation to primitive morphology hypothesis, following phylogenetic tree proposed by McCraken and Sheldon (1998) using osteological characteristics. A) Plesiomorphic condition similar to current shape of Butorides virescens; B) Plesiomorphic condition similar to current shape of Nycticorax nycticorax. Non shaded central ellipse area in adults are in direct proportion to cubic root of body weight and shaded one represent size of the lesser species. All lineal dimensions are in the same scale (approximately 1: 94).

más cercano al ancestro: que la morfología de Butorides sea la condición plesiomórfica o que sea la de Nycticorax. El análisis alométrico de las proporciones, tanto adultas como al eclosionar, permite esclarecer esta situación. Al representar las proporciones corporales con un diagrama transformacional de D'Arcy Thompson simplificado (Fig. 6) se observa que, además de la tendencia al incremento del peso corporal (representado en relación directa con el área del elipsoide que representa el cuerpo en la Fig. 4), en la línea general de las garzas 
existe una tendencia a la elongación en el eje longitudinal (daba por las deformaciones de las caras superior e inferior de los cuadros). Los diagramas muestran las alteraciones en las proporciones a partir de las deformaciones en la rejilla, cuyos vértices (excepto el del extremo del pico) se ubican en las posiciones teóricas según las proporciones en la especie plesiomórfica. Nótese que si se acepta que la forma primitiva fuera semejante a la de Butorides, habría que identificar una explicación para el hecho de que en Nycticorax, Ardea y E. rufescens las longitudes de los picos al nacer (sin haber transcurrido un crecimiento postnatal apreciable) sean menores a los esperables.

\section{DISCUSIÓN}

Una vez aceptada la presencia de procesos heterocrónicos en la evolución del grupo, los datos de crecimiento analizados bajo un esquema filogenético, pueden ayudar a comprender o esclarecer las líneas microevolutivas que siguió este gremio. Para ello, el primer paso es la adopción de una hipótesis filogenética entre las propuestas para el grupo, y afortunadamente los trabajos realizados en este sentido, tanto con caracteres osteológicos, como por hibridización de DNA y por la secuencia de la citocromo b mitocondrial son consistentes entre ellos. En todos, Butorides y Nycticorax son los más primitivos y tienen dos clados hermanos: el que contiene a Bubulcus - Ardea y el de las especies de Egretta. O sea, que existen dos posibilidades lógicas para la selección del morfotipo peramórfico que son estas especies de origen más basal en los árboles, sin embargo, su situación relativa no es esclarecida por estos estudios. Los datos osteológicos tienden a describir como más primitivo a Butorides pero todavía existen puntos controvertidos en estas filogenias. Uno de ellos, por ejemplo, es la ubicación relativa de Bubulcus a quien el análisis osteológico de Payne y Risley (1976) ubicó más cercano al género Egretta que a Ardea, lo cual es contradicho por datos moleculares del citocromo b. Como reflejo de estas incertidumbres, el garzón que previamente pertenecía al género Casmerodius fue cambiado al género Ardea (Ardea alba Linnaeus) (A.O.U., 1998). En el presente estudio, se seleccionó la filogenia propuesta por McCraken y Sheldon (1998), basada en caracteres osteológicos, al considerarla más parsimoniosa.

Como se puede observar, cuando se asume a Butorides virescens como la especie de estructura corporal plesiomórfica (Fig. 6A), la extrapolación de sus proporciones a las demás especies muestra un patrón en el que la tendencia general del grupo fue a incrementar el peso corporal con extensión del periodo de crecimiento, paralelo a lo cual cambian las proporciones corporales por el crecimiento no isométrico del tarso, que aumenta de tamaño en todas, y del pico con alometría positiva en el género Egretta y negativa en Bubulcus y Ardea. Nycticorax sería una línea independiente con un incremento muy marcado de la talla y alometría negativa en las patas y el pico. La segunda hipótesis sería asumir a Nycticorax como morfotipo peramórfico (Fig. 6B) y en este caso Butorides y las demás especies de menor talla corporal habrían evolucionado a partir de un ancestro que representaría una forma de reversión hacia un adulto paedogenético. En este caso, la línea evolutiva general dentro de la familia habría sido la tendencia al alargamiento relativo del pico y del tarso en todas las especies, con alometría positiva. Es decir, esta hipótesis sería consistente con una hipermorfosis gradual que alcanzaría su máxima expresión en Ardea, acentuando la morfología peramórfica. Si se comparan los resultados de ambas hipótesis en el análisis comparativo de las proporciones, tanto en pichones como en adultos, estos apoyan con mayor parsimonia a Nycticorax como morfotipo más primitivo.

El pico y el tarso en Ardeidae son estructuras muy relacionadas con su forma de vida en ecosistemas de humedales, por ello su desarrollo se supone que esté estrechamente controlado desde el punto de vista genético. Así, la marcada disminución de la proporción pico/tarso en las primeras etapas de vida de $B$. virescens reflejan un crecimiento acelerado de las estructuras locomotoras, que según 
Werschkul (1979) tienen un significado adaptativo relacionado con su estrategia reproductiva. Como esta especie es un nidificante solitario, la precocidad de los pichones y su habilidad temprana para moverse puede ser una adaptación defensiva que sustituya la protección relativa, proporcionada por la vida colonial. A esta conclusión también arribaron Cobo et al. (2000) al analizar el crecimiento de Ixobrychus minutus y Ardeola ralloides. Los pichones de esta especie tienen un período de pre-independencia muy breve en relación con las otras: a la semana de edad ya pueden trepar, a los 15 días se mueven libremente de una rama a otra y, potencialmente, puede volar entre los 20 y 25 días.

El crecimiento en las primeras etapas de vida en este grupo tiende a ser más isométrico y las diferencias se acentúan en momentos más tardíos, posiblemente porque a temprana edad es más ventajoso evolutivamente alcanzar una talla superior para sobrellevar las fuentes de mortalidad, que establecer diferencias morfológicas interespecíficas, cuya función ecológica en la segregación de los subnichos ecológicos se alcanzará plenamente mucho más tarde, luego de haber alcanzado la independencia.

En los pichones de esta familia de aves se muestra una marcada similitud morfológica, que disminuye con el crecimiento, lo que dificulta su identificación en ausencia de los parentales. Estas diferencias se evidencian en la comparación de las proporciones pico/tarso que sufren diferencias marcadas en las especies más acuáticas, estando en el otro extremo $B$. ibis sin prácticamente diferencias entre las proporciones en pichones y adultos, lo que evidencia un crecimiento que tiende a ser isométrico en su conjunto.

\section{AGRADECIMIENTOS}

Se agradece el apoyo de los colectivos de estudiantes de la carrera de Biología en el trabajo de campo desarrollado durante los años de estudio, y los acertados señalamientos de los revisores anónimos que contribuyeron a mejorar la calidad del manuscrito final.

\section{RESUMEN}

El orden Ciconiiformes incluye especies de aves zancudas de tamaño mediano a grande, con dinámicas de crecimiento corporal que pueden ser analizadas para esclarecer las tendencias microevolutivas del grupo. Las garzas (familia Ardeidae) provienen de un ancestro común, sin embargo, existen diferencias en la forma corporal de los adultos actuales y se ha sugerido un proceso de evolución por heterocronías. Sin embargo, los estudios de crecimiento previamente se han enfocado solo en las dimensiones lineales y no se han analizado los cambios alométricos. En el presente trabajo se describen los cambios de proporciones corporales durante el crecimiento en siete especies de ardéidos y se analizan bajo una hipótesis filogenética para identificar el patrón morfológico primitivo entre los géneros basales Butorides y Nycticorax. Para ellos se calcularon las proporciones pico / tarso en 353 pichones, medidos entre 1998 y 2006, y se evaluaron sus cambios en relación con la edad y el incremento del peso corporal. Las especies mostraron diferencias marcadas en la magnitud del cambio en las proporciones pico/tarso entre la eclosión y un momento análogo del crecimiento, excepto Bubulcus que tiende a crecer de forma casi isométrica. Los cambios alométricos al crecer conjuntamente con las proporciones al nacer, generan un crecimiento diferencial que produce las disímiles morfologías adultas que se expresan en el grupo de las garzas. La tendencia general de estos cambios es de un incremento ligero en el medio del crecimiento para luego descender a casi la misma proporción inicial. El crecimiento en las primeras etapas de vida tiende a ser más isométrico y las diferencias se acentúan en momentos más tardíos. La hipótesis de asumir a Nycticorax como morfotipo peramórfico es más parsimoniosa en las tendencias de cambio dentro del grupo, resultando en un alargamiento relativo con alometría positiva del pico y del tarso en todas las especies. Esta hipótesis sería consistente con una hipermorfosis gradual que alcanzaría su máxima expresión en Ardea.

Palabras clave: alometría, desarrollo, zancudas, microevolución, heterocronía.

\section{REFERENCIAS}

Acosta, M., Mugica, L., \& Martínez, P. (1990). Segregación del subnicho trófico en seis especies de Ciconiformes. Ciencias Biológicas, 23, 68-81.

Acosta, M., Mugica, L., \& Denis, D. (2002). Dinámica de los principales gremios de aves que habitan la arrocera sur del Jíbaro, Sancti Spiritus, Cuba. El Pitirre, 15(1), 25-30.

American Ornithologists' Union (A. O. U.). (1998). Checklist of North American birds (7th ed.). Washington, D.C.: American Ornithologists' Union. 
Cobo, J., Fouces, V., González-Martín, M., Pedrocchi, V., \& Ruiz, X. (2000). Non-heterochronic developmental changes underlie morphological heterochrony in the evolution of the Ardeidae. Journal of Evolution Biology, 13, 269-276.

Custer, T. W., \& Peterson Jr., D. W. (1991). Growth rates of great egret, snowy egret, and black-crowned nightheron chicks. Colonial Waterbird, 14, 46-50.

Denis, D. (2011). Patrones de crecimiento postnatal en ocho especies de garzas (Aves: Ardeidae). Revista de Biología Tropical, 59(2), 771-787.

Denis, D. \& Ponce de León, J. L. (2006). Ecología reproductiva de la garza rojiza Egretta rufescens (Aves: Ardeidae) en la ciénaga de Birama. Biología, 20(1-2), 17-23.

Denis, D., Rodríguez, A., Rodríguez, P., \& Jiménez, A. (2003). Reproducción de la garza ganadera (Bubulcus ibis) en la ciénaga de Birama, Cuba. Journal of Caribbean Ornithology, 16(1), 45-54.

Denis, D., Mugica, L., \& Acosta, M. (2000). Morfometría y alimentación del aguaitacaimán (Butorides virescens) en las arroceras del sur del Jíbaro. Biología, 14(2), 133-140.

Denis, D., Rodríguez, S., Antunez, O., \& Fortes, H. (2009). Reproduction of green heron (Butorides virescens) in Birama Swamp, Cuba. Journal of Caribbean Ornithology, 22, 83-89.

Freymann, B. P., \& Schuchmann, K. L. (2008). Postnatal growth rates of hummingbirds: review and new records. Wilson Journal of Ornithology, 120, 884-887.

Hudson, J. W., Dawson, W. R., \& Hill, R. W. (1974). Growth and development of temperature regulation in nestling cattle egrets. Comparative Biochemestry and Physics A., 49, 717-741.

Kushlan, J. A. (1977). Diferential growth of body parts in the white ibis. Auk, 94(1), 164-167.

McCracken, K. G., \& Sheldon, F. H. (1998). Molecular and osteological heron phylogenies: sources of incongruence. Auk, 115, 127-141.
Mugica, L., Acosta, M., \& Denis, D. (2003). Variaciones espacio temporales y uso del hábitat por la comunidad de aves de la arrocera sur del Jíbaro, Sancti Spiritus, Cuba. Biología, 17(2), 105-113.

O'Connor, R. J. (1984). The growth and developments of birds. New York: John Wiley and Sons.

Payne, R. B., \& Risley, C. J. (1976). Systematics and evolutionary relationships among the herons (Ardeidae). Miscellaneus Publication, University Michigan Museum Zoology, 150, 1-15.

Raff, A. R. (1996). The shape of life. Genes, development, and the evolution of animal form. Chicago: University of Chicago Press.

Rice, S. H. (1997). The analysis of ontogenetic trajectories: When a change in size or shape is not heterochrony. Proceedings of the National Academy of Science, 94, 907-912.

Ricklefs, R. E. (1973). Patterns of growth in birds II. Growth rate and mode of development. Ibis, 115, 177-201.

Ricklefs, R. E. (1979a). Adaptation, constraint, and compromise in avian postnatal development. Biological Review, 54, 269-290.

Ricklefs, R. E. (1979b). Patterns of growth in birds. V. A comparative study of development in the starling, common tern, and japanese quail. Auk, 96, 1030.

Sheldon, F. H. (1987). Phylogeny of herons estimated from DNA-DNA hibridization data. $A u k, 104,97-108$.

Sheldon, F. H., Jones, C. E., \& McCracken, K. G. (2000). Relative patterns and rates of evolution in heron nuclear and mitochondrial DNA. Molecular Biology and Evolution, 17(3), 437-450.

Siegfried, W. R. (1973). Food requirements and growth of cattle egret in South Africa. Living Bird, 11, 193-206.

Thompson, D' A. W. (1942). On growth and form. Cambridge, U.K: Cambridge University Press.

Werschkul, D. F. (1979). Nestling mortality and the adaptative significance of early locomotion in the Little Blue Heron. Auk, 96, 116-130. 